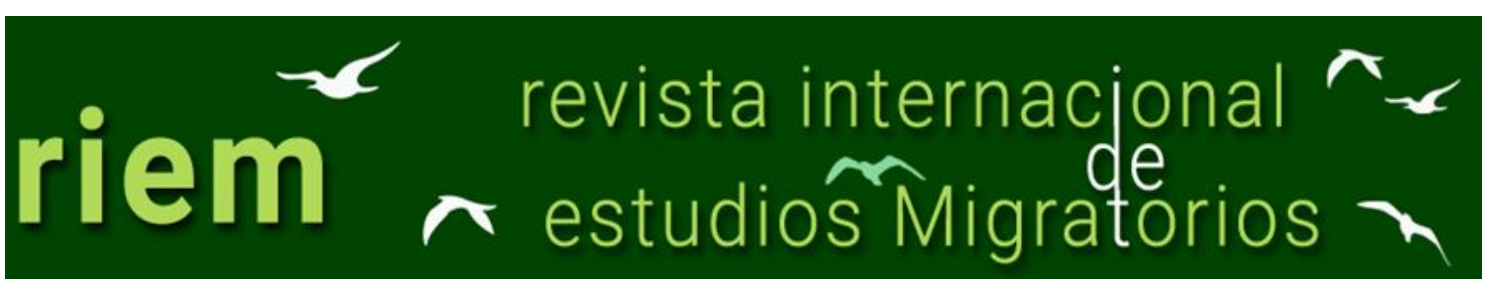

ISSN: $2173-1950$

\title{
Enseñanza de español a refugiados. Marco de referencias y posibles perspectivas
}

Margarita Isabel Asensio Pastor ${ }^{1}$

Resumen: Este trabajo plantea un marco de referencia y posibles perspectivas para la enseñanza-aprendizaje de español como segunda lengua (L2) /lengua extranjera (LE) centrado en la figura social del refugiado. Desde el punto de vista lingüístico, este grupo requiere de la exigencia urgente de aprender el idioma de la sociedad de acogida para poder incorporarse a dicha sociedad. Sin embargo, reconocida esta necesidad, a continuación, surge la cuestión contigua acerca de si ello puede resolverse mediante metodologías convencionales o si, por el contrario, abre un campo nuevo de especificidad. Para ello, tratamos de resolver esta cuestión, procurando atender a todos los posibles ejes condicionantes de esta problemática y, en consecuencia, establecer un marco de referencias que sea de utilidad para proceder a perseverar por esa dirección en el futuro.

Palabras clave: Refugiados, Lingüística Aplicada, Español como segunda lengua (L2) /lengua extranjera (LE), Marco de referencia.

${ }^{1}$ Departamento de Educación, Universidad de Almería, Almería, España. map1o9@ual.es 


\title{
Teaching Spanish to refugees. Framework of references and possible perspectives
}

\begin{abstract}
This work presents a frame of reference and possible perspectives for the teaching learning of Spanish as a Second Language (SL) / Foreign Language (FL) centred on the social figure of the refugee. From the linguistic point of view, this group requires the urgent need to learn the language of the host society to be able to join that society. However, recognizing this need, the next question arises as to whether this can be solved by conventional methodologies or if, on the contrary, it opens a new field of specificity. To do this, we try to resolve this issue, trying to address all possible conditioning factors of this problem and, consequently, establish a framework of references that is useful to proceed to persevere through this direction in the future.
\end{abstract}

Keywords: Refugees, Applied Linguistics, Spanish as a Second Language (SL) / Foreign Language (FL), Frame of reference. 


\section{Introducción}

La enseñanza de lenguas no maternas, como preocupación científica formal y referencia para la lingüística aplicada, arranca a principios de los 60 en Estados Unidos, según la cronología propuesta por Calvo (1999) y Richards y Rodgers (1986 [1998]) con algún precedente aislado en la década anterior (García-Marcos, 2018). Eso no implica que, como actividad social, aprender lenguas distintas de la materna no haya sido una actividad tan antigua como la Humanidad, al menos desde que se dispone de constancia escrita. Incluirla entre las preocupaciones formales de la lingüística, en cambio, es logro relativamente reciente, como hemos expuesto. En ese momento fundacional se pretendía depurar el aprendizaje de idiomas extranjeros por parte de los soldados norteamericanos desplazados en Europa, para lo que se requirió de la ayuda especializada de los lingüistas.

Esas primeras aplicaciones abrieron la puerta a una casuística considerablemente diversificada, de la que entonces solo se estaba ante un primer caso. Además de soldados desplazados, también aprendían lenguas diferentes de la propia los estudiantes en los centros de formación, quienes comerciaban internacionalmente, los científicos que buscaban intercambiar conocimiento, un elenco amplio y variopinto que conformó muy pronto un dominio, el de las lenguas extranjeras. Solo que había otros casos de aprendizaje y/o adquisición de lenguas no maternas. En las sociedades multilingües, los individuos adquieren desde su infancia una lengua diferente de la familiar, incorporada a sus entornos comunicativos ordinarios desde su primera socialización; al respecto, Nussbaum y Unamuno (2014) revisan las perspectivas de la educación plurilingüe en España y América Latina.

Por tanto, esa necesidad social de aprender una lengua puede venir sobrevenida, en especial, a consecuencia de los movimientos migratorios. En este sentido, quien se incorpora a una nueva sociedad, precisa del idioma de acogida para poder integrarse en ella. En consecuencia, pronto aparece un nuevo dominio, el de las segundas lenguas (a partir de ahora L2), que ya a mediados de los años 80 cuenta con su propio epígrafe dentro de la bibliografía especializada y con referencias básicas (Larsen-Freeman y Long, [1991] 1994). Por tanto, desde ese momento, se ha asistido a una continuada subespecificación de áreas dentro de estas enseñanzas que ha supuesto, no solo una redefinición parcial de su conceptualización, sino también el desarrollo de estrategias específicas de enseñanza.

Así pues, la enseñanza de lenguas no maternas comienza a clasificarse en dos tipos: enseñanza de lenguas extranjeras y enseñanza de segundas lenguas. Aunque existe un 
evidente denominador común y compartido entre ambas, lo cierto es que cada supuesto de enseñanza tiene una singularidad muy marcada materializada en la metodología.

En los últimos tiempos, esa parte de la lingüística aplicada ha de responder a una figura social que, no desconocida en las sociedades europeas, sí que ha incrementado de manera muy notable su presencia en relación con etapas anteriores. Siempre ha habido refugiados en Europa, aunque nunca tantos, tan extendidos por todo el continente, tan heterogéneos en su procedencia ni tan marcados por aspectos geopolíticos y sociales en un mundo globalizado. En lo lingüístico, de inmediato, plantean la necesidad de adquirir las lenguas propias de las sociedades de arribada, así como si esto puede resolverse mediante metodologías convencionales o si, por el contrario, abre un nuevo campo de especificidad. Esa es la pregunta que trata de resolverse desde este trabajo, procurando atender a todos los posibles ejes condicionantes de esa problemática, con la pretensión de establecer un marco de referencias que sea de utilidad para proceder a perseverar en esa dirección.

\section{El contexto (I). La sociología de los movimientos demográficos contemporáneos}

El notable incremento de los migrados, con cifras sin precedentes en la historia de la Humanidad, es una de las principales características que definen la actual situación geopolítica en el mundo. En concreto, a raíz de la llamada "crisis de los refugiados en Europa" (Spindler, 2015) en 2015 se alcanzó los 244 millones de personas desplazadas Organización de las Naciones Unidas (ONU, 2016); actualmente ascienden a 272 millones (ONU, 2019). Esos desplazamientos, desde luego, obedecen a causas diversas y heterogéneas. Una parte de ellos puede ser considerada fruto de una elección libre y voluntaria, al menos en cierta medida (Posada, 2009). Otras, sin embargo, son consecuencia directa de necesidades perentorias, de nuevo notablemente diversificadas, que irían desde la aspiración a una mejora de condiciones precarias de vida hasta necesidades inmediatas de supervivencia y protección en otro lugar, pasando por desplazamientos como consecuencia de factores demográficos (tránsito desde sociedades con alta natalidad a sociedades con baja natalidad) o por desastres naturales; en ocasiones, ese viaje migratorio se realiza a países donde ya existe una red social establecida de familiares o amigos/conocidos (Organización Internacional para las Migraciones [OIM], 2013).

En concreto, el grupo integrado por quienes precisan protección para su supervivencia lo constituyen los refugiados cuyo estatus queda definido por la Convención de Ginebra 
en su Estatuto de los Refugiados (1951) en el Artículo 1. Sección A. 2. como una persona que:

Debido a fundados temores de ser perseguida por motivos de raza, religión, nacionalidad, pertenencia a determinado grupo social $u$ opiniones políticas, se encuentre fuera del país de su nacionalidad y no pueda o, a causa de dichos temores, no quiera acogerse a la protección de tal país; o que, careciendo de nacionalidad y hallándose, a consecuencia de tales acontecimientos, fuera del país donde antes tuviera su residencia habitual, no pueda o, a causa de dichos temores, no quiera regresar a él (p.2).

En esa misma línea, y todavía dentro del ámbito internacional, el Protocolo sobre el Estatuto de los Refugiados (ONU, 1967) firmado en Nueva York mantiene la definición anterior, aunque introduce los siguientes cambios en el Artículo I.2.

A los efectos del presente Protocolo y salvo en lo que respecta a la aplicación del párrafo 3 de este artículo, el término "refugiado" denotará toda persona comprendida en la definición del artículo 1 de la Convención, en la que se darán por omitidas las palabras "como resultado de acontecimientos ocurridos antes del $1 .^{\circ}$ de enero de 1951 y..." y las palabras "...a consecuencia de tales acontecimientos", que figuran en el párrafo 2 de la sección A del artículo 1 (p.2).

España desarrolló su propia legislación particular, ajustada a las directrices anteriores, mediante la Ley 12/2009 que aparece en el BOE de 30 de octubre de 2009 (Jefatura del Estado, 2009). En ella se define al refugiado como aquella persona que está fuera de su país por temor a persecuciones por causas políticas, de raza, religión, nacionalidad, pertenencia a un grupo social o de género. La norma española incluye también la categoría de apátrida, definido como quien carece de nacionalidad y se encuentra en las situaciones anteriormente mencionas que le imposibilitan poder volver a su país.

Una parte considerable de la población desplazada en el mundo actual entra dentro de la categoría de apátrida. Según Tendencias Globales, informe presentado por el Alto Comisionado de las Naciones Unidas para los Refugiados (ACNUR) en 2017, hasta el 31 de diciembre del año anterior habría 65,6 millones de personas desplazadas, de las que 22,5 millones serían refugiados (17,2 bajo el auspicio directo de ACNUR; 5,3 registrados por el UNRWA). De esas cifras se generan otras igualmente relevantes de la problemática 
que se está abordando, como los 2,8 millones de solicitantes de asilo y los aproximadamente 10 millones de apátridas; exponentes todos ellos de la envergadura internacional de esta cuestión.

\section{El contexto (II). La situación española.}

Es conocido que España ha cambiado drásticamente su tendencia respecto a los movimientos migratorios. Como señalan Valero-Matas et al., (2014), entre 1995-2007 se produjo una etapa de aceleración migratoria con un notable incremento de la población extranjera en este país. Al respecto, los últimos datos ofrecidos por la OIM, sitúa a España en el séptimo puesto de los principales países de destino de las migraciones internacionales (OIM, 2019).

Este hecho supone, por tanto, que España haya pasado de ser un país tradicionalmente exportador de emigración, a convertirse en centro receptor de población foránea, fundamentalmente debido al crecimiento económico experimentado a partir de la década de los 90 del siglo pasado (Alted, 2008). Ello tuvo consecuencias lingüísticas inmediatas, generando un profuso contacto de lenguas, por un lado, y, por el otro, la necesidad de aprender las lenguas de España en función de la ubicación del migrado (Carmona y GarcíaMarcos, 2002; García-Marcos, 2002, 2005, 2017; García-Mateos, 2004; Feuernherm y Ramanathan, 2015; Asensio y Carmona, 2019). En consecuencia, la demanda de formación lingüística en segundas lenguas.

Ahora bien, el movimiento migratorio ha tenido también su correspondiente cuota de refugiados. De acuerdo con datos oficiales del Ministerio del Interior de España (en línea), se observa un incremento exponencial en las solicitudes de protección internacional en España a partir de 2015 y que sigue al alza como se muestra en el siguiente gráfico (Figura 1) que recoge la tendencia de los últimos catorce años. 


\section{Figura 1. Evolución del número de solicitantes de protección internacional en España (2006-2019)}

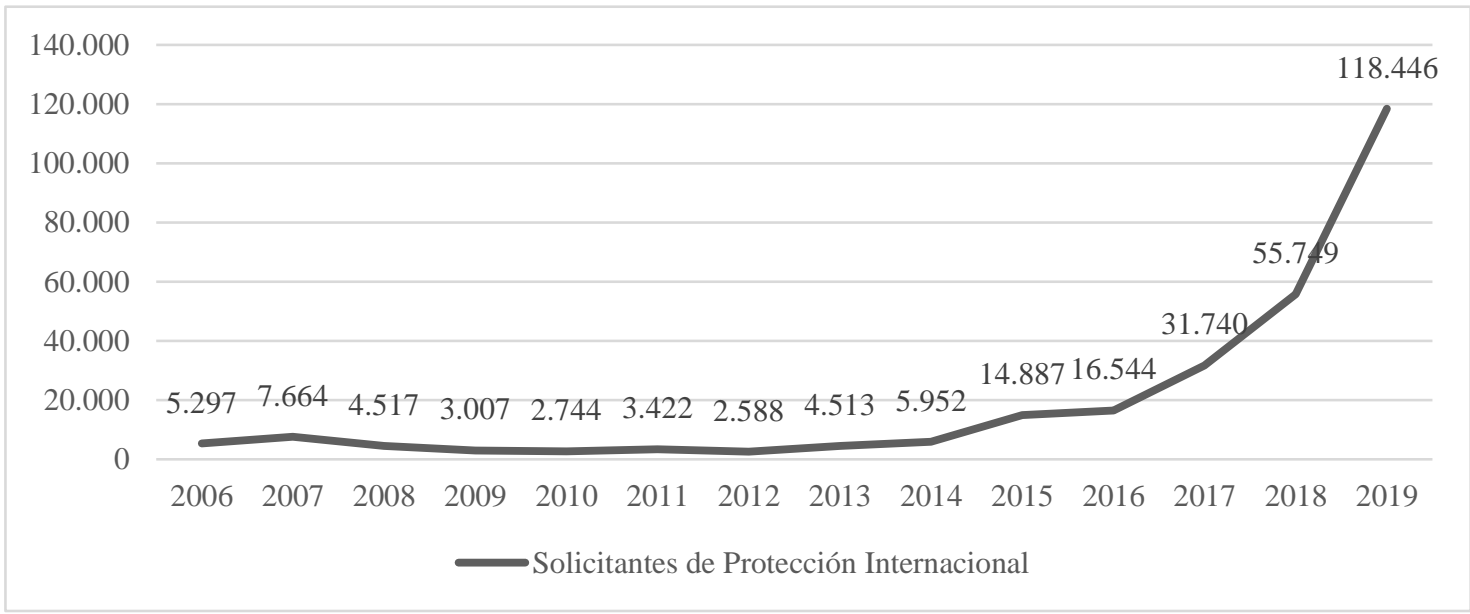

Nota. Adaptado de datos e información estadística del Ministerio del Interior (en línea)

Hay, por lo tanto, un sector de población significativo, progresivamente más presente en la sociedad española que responde a ese epígrafe. Por tanto, desde el punto de vista lingüístico, este grupo requiere de la exigencia urgente de aprender el idioma de la sociedad de acogida para poder incorporarse a dicha sociedad. Ahora bien, reconocida esta necesidad, surge la cuestión contigua que se plantea en este artículo y es si la enseñanza de español LE a refugiados constituye un campo mínimamente sustantivo de aplicaciones en esta dirección. En consecuencia, la urgente obligación de constituir un marco referencia y ofrecer posibles actuaciones metodologías ante la especificidad que pueda presentar el aprendiente de español como lengua de acogida al refugiado.

\section{La bibliografía sobre enseñanza de lenguas extranjeras a refugiados}

La bibliografía especializada tampoco aporta orientaciones definitivas, o cuando menos claramente delimitadas respecto, del caso concreto de la enseñanza de lenguas a refugiados (Raga y Ortí, 1997; Villalba y Hernández, 1999; García-Mateos, 2003, 2004; García-Marcos, 2003; García-Parejo, 2003, 2004; Muñoz-López, 2004; Jiménez, 2015; Asensio y Carmona, 2019). En el estudio de Keyes y Kane (2004) sobre la salud mental de refugiados bosnios en Estados Unidos en donde se concluyó, entre otras cuestiones, que uno de los factores que promuevan la pertenencia y la adaptación exitosa de dichos refugiados era el poder desenvolverse en la lengua del país de acogida. Por otra parte, el aprendizaje de la lengua de acogida se convierte en un requisito legal; tal es el caso que recoge Elmeroth (2011) relativo a los refugiados en Suecia que deben pasar un examen en 
esta lengua para poder acceder al mercado laboral. Asimismo, no son pocos los países que exigen competencia comunicativa certificada en la lengua oficial de esos países para obtener la ciudadanía o, en última instancia, la nacionalidad como es el caso de España (Jefatura del Estado, 2015).

En ese sentido se encontrarían en una situación similar a la de los inmigrados, sobre los que hay una prolija atención en la política lingüística de los países occidentales. En García-Marcos (2002) se rinde cuenta de las principales soluciones adoptadas por los estados occidentales, de su rendimiento social y hasta de las reservas éticas que pudieran formularse.

Sin embargo, en el caso de España no existe una política lingüística nacional que preste atención lingüística al migrado, cuestión que ya señaló Salazar (1998) en los 90 y que todavía sigue vigente, como sí sucede en otros países con mayor tradición migratoria como Gran Bretaña que tiene en marcha un plan nacional, desde 1960, bajo el nombre de Linguistic Minorities Project (Mateo, 1995: 118). No obstante, en España sí existen políticas lingüísticas autonómicas bastante explícitas en esa dirección.

En el caso de Andalucía Asensio y Carmona (2019) indican que la enseñanza reglada y pública de español a adultos refugiados e inmigrantes se canaliza a través de dos vías:

- Los Centros y Secciones de Educación Permanente (CEPER y SEPER), creados mediante el Decreto 196/2005, de 13 de septiembre, en los que se imparten enseñanzas básicas, que abarcan desde la alfabetización funcional hasta la preparación para la obtención de la titulación básica y acceso a otros niveles educativos.

- Las Escuelas Oficiales de Idiomas (en adelante EEOOII), enmarcadas en las Enseñanzas de Régimen Especial, en el artículo 60.2 de la Ley de Educación 2/2006, de 3 de mayo, donde se puntualiza que: "las escuelas oficiales de idiomas fomentarán especialmente el estudio de las lenguas oficiales de los Estados miembros de la Unión Europea, de las lenguas cooficiales existentes en España y del español como lengua extranjera”. En Andalucía, las EEOOII, reguladas por el Decreto 15/2012, de 7 de febrero, por el que se aprueba el Reglamento Orgánico de las Escuelas Oficiales de Idiomas en la Comunidad Autónoma de Andalucía.

Luego de trabajos pioneros como el de Miquel (1994, 1995) o Mateo (1995), la enseñanza del español a inmigrantes se ha ido incorporando a la nómina de la lingüística aplicada española, de manera quizá pausada, aunque constante. Prioritariamente centrada en la enseñanza a adultos, (Hernández y Villalba, 2003a, 2003b, 2005; 
Larrañaga, 2003; Pozzo, 2009; Fernández, 2009; Níkleva, 2014, 2017; García-Flores, 2014; Bravo-García, Gallardo-Saborido, Santos de la Rosa y Gutiérrez, 2014; Carmona, 2018; Asensio y Carmona, 2019) o niños y adolescentes (Ojeda, 2009; Villarrubia, 2009; Herrera, 2016; Asensio, 2016, 2018), entre otros. Tampoco faltan panorámicas más genéricas, como los repertorios bibliográficos críticos sobre sociolingüística e inmigración (García-Marcos, 2002) o adquisición de segundas lenguas (Martín, 2004; Salazar, 2004).

Cabe mencionar, por otra parte, que cuando se ha prestado atención a los refugiados, se los ha integrado sin mayores matices dentro de la categoría de inmigrantes (Miquel, 1994, 1995; Villalba y Hernández, 1995, 2000, 2002, 2010; Villalba, Hernández y Aguirre 2001; García-Ortiz y Khraiche Ruiz-Zorrilla, 2013). Este realmente es el punto central de la reflexión que se plantea aquí. Tal y como ya se ha avanzado, se trata de discriminar si existen razones suficientes, o no, para establecer un campo mínimamente sustantivo de aplicaciones en esta dirección.

\section{Hacia un perfil sociolingüístico del refugiado}

\section{1. $\quad$ Expectativas de aprendizaje/adquisición de la lengua meta}

Todo aprendizaje/adquisición de una lengua no materna trae consigo aparejado un conjunto de expectativas y actitudes que condicionan de manera determinante su desarrollo. Slagter (1994) propuso clasificarlas, siguiendo una metodología estandarizada en sociolingüística, a partir del análisis de la construcción de los tests de lengua (concretamente su fiabilidad y validez) y de las noticiones de competencia y enseñanza comunicativa inherentes. Así, el esperanzador éxito de sus primeros resultados no bastó para seguir con la impronta empírica que subyacía en su propuesta. Sí que, por el contrario, se ha preferido una opción más teórica y especulativa para abordar esta temática. Eso ha abierto la puerta a discusiones, más o menos apriorísticas, acerca de qué diferencias había en las motivaciones de estudiantes extranjeros y de segundas lenguas, cómo repercutía en la cotidianidad de esos escolares, qué función social desempeñaban esos aprendizajes o cuál era la influencia de los entornos institucionales entre los que se registraban esos procesos (Muñoz, 2002; Díaz-Corralejo, 2001).

De este modo, si se tienen en cuenta estas consideraciones, pueden extraerse dos conclusiones muy amplias, esta vez sí, de casi inmediata corroboración empírica. De un lado, hay una motivación tendencialmente distinta en estudiantes de lengua extranjeras y aquellos otros que incorporan una segunda lengua. Tampoco estaría de más precisar en qué se concreta con exactitud esa diferenciación que se da por sentada, pero en la que 
paradójicamente la bibliografía no se ha adentrado, convirtiéndola en un postulado en su práctica disciplinar. De otro, en el caso de las segundas lenguas, esos procesos de adquisición desempeñan un papel determinante en la socialización del individuo y, en consecuencia, en el desarrollo de toda su actividad vital en la comunidad de acogida. Son, sin duda, la puerta que abre (o cierra) la integración de los inmigrados.

Los refugiados, es obvio, se encuentran dentro del segundo gran grupo, es decir, del determinante en la socialización, con expectativas y necesidades comunicativas, como mínimo, análogas a las de otras personas migrantes, aunque motivados por la idiosincrasia de sus desplazamientos forzosos. En el estudio exploratorio de Asensio y Carmona (2019), se traza un perfil de estudiante refugiado en España concretado en tres rasgos principales: la historia personal, esto es, personas procedentes de situaciones traumáticas; la heterogeneidad de estos en cuanto a nacionalidad, edad, estilos de aprendizaje, formación o profesión, entre otros y, por último, la situación administrativa de estos en España.

Para los refugiados, el desplazamiento no es una opción voluntaria y, por tanto, con retorno potencial y libre en el momento que se estime oportuno. El refugiado precisa con mayor énfasis integrarse en un entorno social que no es exactamente de acogida, sino más bien de protección. Por decirlo de manera gráfica, el emigrante se va de su lugar de origen, el refugiado huye. Todo ello debe incidir de manera muy significativa en las expectativas de adquisición de estos hablantes, en función de lo que son unas necesidades de socialización absolutamente propias y diferenciadas de las de otros grupos foráneos. Bien es verdad que hasta que no se disponga de datos empíricos al respecto, solo cabe moverse con prudencia y siempre dentro del dominio de las hipótesis.

\subsection{Las estructuras de acogida}

Gran parte de esas necesidades diferenciadas arranca del tipo de incorporación a la sociedad de acogida que se haya producido en cada caso. La inmigración se rige por una casuística muy heterogénea que oscilaría, en sus extremos, desde las migraciones individuales hasta las redes de relación que conforman el llamado efecto llamada. En todos sus supuestos, no obstante, se mantienen algunos rasgos comunes bastante estables: los inmigrantes se integran en comunidades de migrados del mismo origen, con la consiguiente propensión a la ghettizzación, en las que la lengua de origen resuelve numerosas situaciones comunicativas inmediatas, al menos dentro de ese contexto. La diglosia resultante puede llegar incluso a institucionalizarse con el paso del tiempo, como de hecho ha sucedido en Australia con bastantes minorías de origen inmigrado (GarcíaMarcos, 1999). En todo caso, se trata de procesos abiertos, sujetos a la evolución de la 
dinámica social de cada comunidad (Fernández, 2009; García-Marcos, 2017; Naeb, Young-Scholten y Sosinski, 2019).

En el caso de los refugiados, abundan en mayor medida los desplazamientos individuales, o en grupos reducidos. Además, estos suelen estar regulados por legislación e infraestructura administrativa bastante explícitas o, lo que es lo mismo, se produce lo que se conoce como recepción programada. En el caso de España, se integran dentro de establecimientos públicos, los llamados Centros de Acogida a Refugiados (CAR), que cuentan con una considerable cobertura legal y están fundamentalmente regulados por el artículo 264 del Reglamento de la Ley Orgánica 4/2000 (derechos y libertades de los extranjeros en España y su integración social), aprobado mediante Real Decreto 557/2011 (20-III-2011) (Ministerio de la Presidencia, 2011). En tanto que servicio público, esos centros dispensan una amplia gama de prestaciones sociales: alojamiento y manutención, asistencia psicológica, servicios de integración social (educación sanidad, etc.), gestión de ayudas económicas, orientación profesional y de estudios, ocio e incluso formación idiomática.

La enseñanza de lenguas a refugiados dispone ya, como sucede en el ejemplo español, de infraestructura formal y jurídica ostensiblemente más regulada que la de inmigrados.

Así, desde el punto de vista formal y jurídico, los refugiados en España deben recibir formación mínima en la lengua o lenguas del Estado español como sucede con los CAR que ofrecen posibilidades latentes y un primer embrión de atención idiomática. El problema reside en que no está regularizado el qué y el cómo, así el documento El español como nueva lengua (Instituto Cervantes, 2005) constituye uno de los pocos que atiende a las necesidades de los aprendientes migrantes. Como puntualizan Asensio y Carmona (2019), se debe enfatizar las adaptaciones curriculares más pertinentes y adecuadas para la inclusión de este colectivo, estableciendo un equilibro entre los diseños curriculares externos, fijados por el currículo, y los internos, surgidos en el aula.

Por tanto, haría falta complementar con decisiones de política lingüística explícita en esa dirección, fijando con claridad la adquisición de la lengua de recepción como un objetivo claro dentro de sus actividades. Por supuesto, ello debería llevar acompasado de inmediato el correspondiente desarrollo mediante estrategias concretas de planificación lingüística, concentrada específicamente en esas enseñanzas.

Así pues, la principal estrategia didáctica orientada a la enseñanza de español a refugiados, como sucederúa en otros contextos, es crear un clima de confianza y 
comprensión en el aula, aunque especialmente delicado por la propia historia personal. En segundo lugar, el docente, como organizador, guía o dinamizador debe realizar una adecuada selección, adaptación y secuenciación de materiales y contenidos en el aula. Asimismo, en este contexto, este habrá de trabajar la resiliencia de este alumnado, sobre todo, en los casos más extremos.

\subsection{El estrés postraumático de los refugiados}

La bibliografía suele ser también unánime a la hora de señalar que los refugiados pueden presentar lo que se conoce con el término de estrés postraumático (Kleinmann, 1984; Corvo y Peterson, 2005; Carlsson, Mortensen y Kastrup, 2006; Hayward, 2007; Benseman, 2012). A él cabría añadir el llamado Síndrome de Ulises (Achotegui, 2000). El primero se produce a consecuencia de una situación traumática relacionada con la vida y la muerte. El segundo tiene que ver con la vivencia de huida del país de origen y el periplo de desplazamiento hasta la sociedad de destino.

En todo caso, suele ser común vincular de nuevo la experiencia del refugiado con la migratoria. Para autores como Kleinmann (1984, p. 210), toda migración, incluyendo la voluntaria, es una experiencia traumática, habida cuenta de que se produce un desajuste vital, social e identitario al abandonar a su comunidad. De esa manera se establecería una especie de escala de estrés² (Adkins, Sample y Birman 1999; Hayward, 2007) que incluiría tres estadios:

1. Estrés migratorio provocado por el viaje en sí mismo hacia otro lugar.

2. Estrés cultural o estrés aculturativo al enfrentarse, no solo a una nueva lengua, sino a una nueva cultura, nuevas costumbres, nuevas formas de relación social, etc.

3. Estrés traumático como resultado de la causa que hace abandonar su hogar al refugiado o las vicisitudes por las que atravesó hasta poder abandonar su hogar como puede ser haber sufrido tortura.

El correspondiente a los refugiados se situaría en el máximo de esa escala y se correspondería con lo que se caracteriza como TEPT (Trastorno de Estrés Postraumático), Carlssonn et al., 2006; American Psychological Association. [APA], 2011) definido por los siguientes rasgos:

\footnotetext{
${ }^{2}$ Por estrés entendemos el desequilibrio entre las demandas ambientales percibidas y las capacidades de respuesta del sujeto (Achotegui, 2005: 1).
} 
- Recuerdos angustiosos, recurrentes, reacciones disociativas (sufrir flashbacks), problemas para dormir, pesadillas y cansancio.

- Evitación o esfuerzo para evitar los recuerdos traumáticos.

- Alteraciones negativas cognitivas y del estado de ánimo: depresión baja motivación e interés, baja autoestima, problemas de concentración y/o atención, facilidad para distraerse.

- Problemas con la autoridad.

Es cierto que este último listado probablemente sea susceptible de ser ampliado. Estos trabajos apuntan a que el refugiado limita, restringe, o en todo caso monitoriza, sus relaciones con personas procedentes del entorno que ha abandonado. En todo caso, parece evidente que su perfil psicológico medio no se corresponde ad hoc con el del inmigrado estándar y que, por consiguiente, pueden desenvolverse entre coordenadas relativamente distintas que condicionen parte de sus procesos de aprendizaje.

\subsection{El resto de los factores condicionantes en la enseñanza de lenguas extranjeras}

En los procesos de adquisición/aprendizaje de lenguas extranjeras es previsible que intervengan el resto de los factores pertinentes entre otros grupos de hablantes. En primer lugar, y como por otra parte era previsible, la edad puede condicionar de manera significativa esos procesos de adquisición. La bibliografía hace tiempo que abandonó el tan manido tópico de la irreversibilidad de los condicionamientos psicológicos vinculados a la edad de aprendizaje lingüístico, de poca base en la actualidad. (Santos, 1999; Muñoz, 2002; Salazar, 2004, Garcia-Marcos, 2018). Pero sí que es cierto que la edad puede actuar asociada con otros factores considerablemente activos. De esa manera, los niños y jóvenes suelen focalizarse más que los adultos en los procesos de enseñanza, precisamente porque carecen de otras preocupaciones vitales que sí tienen los segundos. Sin embargo, puede que no hayan desarrollado una formación básica en su país de origen, o que procedan de contextos multilingües y, por tanto, estén familiarizados con el desarrollo simultáneo de varias competencias comunicativas en su propia cotidianidad.

Por una parte, el género es otro eje que ha de ser contemplado, como siempre, no porque introduzca diferencias biológicas debido al sexo, sino por la posibilidad de comportar estereotipos sociales vinculados a hombres y mujeres. Para empezar, el género suele determinar el grado de formación de los estudiantes. En bastantes sociedades, los alumnos suelen recibir más escolarización que las alumnas, con lo que también disponen de mayor porcentaje de estudios superiores. Los datos del Tercer informe mundial sobre el aprendizaje y la educación de adultos de la Organización de las Naciones Unidas para 
la Educación, la Ciencia y la Cultura (UNESCO; 2017) al respecto son determinantes. De los 758 millones de adultos con dificultades para leer y escribir, las mujeres representan dos tercios, o lo que es lo mismo, están en torno a 479 millones de personas. Por otra parte, en diferentes contextos socioculturales el género puede determinar necesidades comunicativas más concretas. Por acudir a un ejemplo ilustrativo, las mujeres dedicadas al hogar en sus países de origen probablemente tendrán demandas lingüísticas relativas al espacio privado (el hogar) y los hijos (crianza, educación y sanidad). Asimismo, habría que tener que encuentra que algunas de ellas proceden de sociedades con esa construcción y delimitación de roles sociales, por lo que se podrían dar en el aula dinámicas que reprodujeran esos mismos modelos, como inhibición cuando se encuentra en grupos mixtos.

El grado de instrucción aporta el tercer factor en la bibliografía (Miquel, 1994, 1995; Santos, 1999; Muñoz, 2002; Salazar, 2004, Garcia-Marcos, 2018). De un lado, el ascenso en la pirámide formativa corre paralelo al de automatismos y destrezas en el aprendizaje/adquisición de otras lenguas. La experiencia previa en el aprendizaje de una lengua extranjera en el sistema educativo resulta altamente positiva para el eventual despliegue de competencias comunicativas posteriores. Por otro lado, el grado de instrucción influirá en las demandas formativas de los alumnos, así como a las de contenidos lingüísticos, culturales y pragmáticos. Igualmente puede afectar a múltiples aspectos de la dinámica de aula, empezando por los estilos de aprendizaje que puedan aplicarse y continuando por el ritmo de las sesiones docentes, el uso de metalenguaje, la preferencia por destrezas y habilidades complejas, las expectativas sobre la práctica docente, la demanda de unos materiales didácticos concretos, etc.

Naturalmente, la lengua de procedencia introduce condicionamientos de considerable peso. Lo hace, además, a dos niveles. En primer lugar, la distancia tipológica entre la lengua de origen y la lengua meta resulta determinante para las nuevas adquisiciones lingüísticas. La secuencia de aprendizaje se acelera en las próximas, pero la de discriminación última se fortalece con la distancia tipológica. Es más fácil empezar a aprender una lengua familiarmente próxima, pero sin embargo los últimos estadios elevados de la lengua meta son más propicios desde la distancia interlingüística que evita interferencias de todo tipo. En segundo lugar, puede resultar igualmente decisiva la escritura, no solo en cuanto a la su dirección y grafemática, sino también al nivel conocimiento metalingüístico que se tenga de esta. En dirección análoga, Bigelow, Vanek, King y Adi (2017) recuerdan que es necesario contar con el sustrato de procedimientos y estilos de aprendizaje importados de los países de origen. 
Existen, por descontado, otros factores que también pueden condicionar, en grado variable, estos procesos, caso del grado y tipo cognitivos del alumnado, sus ya mencionadas necesidades y expectativas o el bagaje cultural y las experiencias del pasado de las que parta. Con todo, hay dos hechos que sí son atribuibles a la posible singularidad de la enseñanza a refugiados. La carga de heterogeneidad, consustancial a toda enseñanza de lenguas no maternas, se multiplica exponencialmente en este supuesto y, sobre todo, es complicado e infrecuente amortiguarla, excepto al inicio de su acceso a los países de acogida. En lenguas extranjeras no es inhabitual operar con grupos de la misma procedencia, dentro incluso de programas previamente establecidos y regulados. La enseñanza de segundas lenguas se dispersa considerablemente más, aunque mantiene latente la posibilidad de confeccionar grupos con una cierta uniformidad en función de sus zonas o lenguas de procedencia. El caso de los refugiados es más complejo debido a su propia naturaleza. Proceden de emplazamientos mucho más aislados, en ocasiones individuales, con asentamientos dispersados, en los que coinciden con refugiados de otras zonas. Es cierto que durante su primera estancia en los centros de acogida existe la posibilidad de trabajar con grupos lingüísticamente homogéneos. Pero esa no deja de ser una solución provisional que, a medio plazo, ha de conducir a un inicio de integración en la sociedad de recepción, entre grupos más amplios y, por consiguiente, más diversificados lingüísticamente. A ello se agrega una segunda circunstancia, como es la intermitencia en su incorporación a las comunidades de acogida (García-Ortiz y Khraiche, 2013). Eso significa que, aún en el supuesto de que se formaran grupos de la misma procedencia social y lingüística, estarían compuestos por alumnos con distinto tiempo de llegada y, naturalmente, con diferente grado de adquisición de la lengua de acogida.

En relación con los refugiados conviene hacer dos puntualizaciones importantes. Ninguno de los anteriores factores es consecuencia directa ni resultante privativa de la situación de refugiado. Son factores universales que condicionan la actuación lingüística, en sentido amplio y abarcador, desde los procesos de adquisición hasta la estratificación social de las lenguas. Además, no intervienen de manera uniforme entre ese colectivo, no existe un condicionamiento de género propio de la categoría refugiado, por ejemplo, sino que están condicionados y actúan en función del origen. Por ejemplificarlo, se podría considerar en este sentido que una mujer ucraniana y una siria tienen poco en común, más allá de compartir sexo biológico. 


\section{La (posible) singularidad de los estudiantes refugiados. Perspectivas y precauciones.}

A la vista de todo lo anterior, podrían perfilarse tres rasgos principales que delimitarían el supuesto migratorio en la enseñanza de lenguas no maternas, directamente vinculados a su historial personal, a sus expectativas y a la dinámica de aula que genera su incorporación. Desde luego, hay que tener muy presente que se trabaja con personas procedentes de situaciones diversas e incluso, a veces, posiblemente traumáticas. Justo por ello, el estudio de una lengua extranjera, la de la comunidad de acogida, puede resultar beneficioso, desde el momento en que facilitará su socialización, así como el desarrollo de su autoestima, aumentando de esa forma su resiliencia, su capacidad para superar circunstancias traumáticas (Capstick y Delaney, 2017). Todo ello se traducirá en una dinámica de aula especialmente cuidadosa con todos aquellos elementos que puedan activar factores negativos, en especial los que puedan mermar esa capacidad de resiliencia que acaba de mencionarse. Del mismo modo, se potenciará en el desarrollo del trabajo docente una actitud positiva y receptiva de las particularidades que este colectivo pueda incorporar al aula y contribuya en su acceso a la sociedad de acogida.

Más allá de eso, realmente es complicado encontrar elementos claramente sustanciales del supuesto vinculado a los refugiados. Por supuesto que los diseños curriculares quedan por completo al margen de este tipo de condicionamientos. Los contenidos nocionales o funcionales no varían en función de la clase de alumnado que va a recibirlos; Los pioneros trabajos de Miquel $(1994,1995)$ ya dejaron patentes las ventajas de una programación de corte más funcional, basada en actos de habla, para atender a las necesidades comunicativas de este colectivo. La cuestión girará en torno a acomodar su secuenciación, adaptar los objetivos, seleccionar materiales específicos, proponer syllabus con énfasis en la particularidad del fenómeno y, sobre todo, articular dinámicas de aula exentas de las marcas negativas que se han apuntado a lo largo de este trabajo.

Tampoco impone, ni tan siquiera recomienda, una metodología particularmente adecuada a este caso. En términos generales se propondría el paradigma comunicativo, en todas sus variantes, el más recomendable en principio para cualquier supuesto de enseñanza de lenguas, simplemente por abarcar la totalidad de la competencia comunicativa y poner el acento en las destrezas y habilidades del aprendiz, a diferencia de otros modelos. Por otra parte, esa parece ser la práctica imperante en las sociedades donde se ha prestado atención explícita y formal a la formación lingüística de refugiados. Tanto el British Council como el Goethe Institut, organismos públicamente implicados en la 
integración lingüística de los refugiados, sin embargo, no reservan cursos específicos para ello.

\section{Reflexiones finales}

Con todos los argumentos expuestos en este artículo, sostenemos que la enseñanza de español a refugiados, más allá de abrir un nuevo epígrafe en el campo de la lingüística aplicada, debe ser encuadrada como un supuesto puntual dentro de la adquisición de segundas lenguas. En este sentido, no hay diferencias determinantes en cuanto al núcleo fundamentalmente lingüístico de estas aplicaciones. No estaría justificado modificar los diseños curriculares, dado que no se generan procesos de interlengua distintos ni, por supuesto, se modifican los condicionamientos tipológicos de las lenguas implicadas en la adquisición/aprendizaje. Por ejemplo, las diferencias entre árabe estándar y español son las mismas, con independencia de que los alumnos sean inmigrados magrebíes o refugiados sirios, aunque es sabido que el árabe presenta una situación diglósica, multiglósica o continuum diglósico (Ferguson, 1959; Blanc, 1960; Badawi, 1973). Por lo tanto, desde este punto de vista, abogamos por una ubicación dentro de los programas de adquisición de segundas lenguas. Naturalmente, ello no significa desentenderse, ni de sus condicionamientos dentro del aula, ni de sus posibles repercusiones fuera de ella. No son elementos contradictorios.

La bibliografía insiste en enfatizar el rol que puede desempeñar la adquisición de la lengua de acogida, en tanto que estímulo para superar los traumas psicológicos de los refugiados, al tiempo que potenciaría su resiliencia (Chadwik y Awad, 2016; Capstick y Delaney, 2017; Finn, 2010; APA, en línea; Utria et al., 2015). Por ello, el componente lingüístico ha de incluirse sistemáticamente en los programas de integración de estos grupos sociales (Feuernherm y Ramanathan, 2015). Así, nada de ello justifica salirse del ámbito de las segundas lenguas. Es más, podría ser hasta contraproducente, formando grupos especiales de alumnos refugiados, en lo que no dejaría de ser una forma de segregarlos de otros hablantes, de guetizarlos dentro del gueto. Muy probablemente la integración empiece, o pueda empezar, compartiendo aula con otras personas que, por otros motivos, han terminado llegado al mismo lugar y teniendo urgencias no tan distintas en muchos aspectos. 


\section{Referencias}

Achotegui, J. (2000). Los duelos de la migración: una perspectiva psicopatológica y psicosocial. En E. Perdiguero y J. M. Comelles (comp.) Medicina y cultura (pp. 88-100). Barcelona: Ediciones Bellaterra.

Achotegui, J. (2005). Estrés límite y salud mental: el síndrome del inmigrante con estrés crónico y múltiple (Síndrome de Ulises). Revista Norte de salud mental de la Sociedad Española de Neuropsicología, 5(21), 39-53.

ACNUR (2017). Tendencias globales de 2016.

https://www.acnur.org/recursos/estadisticas/tendencias-globales-2016/

Alted Vigil, A. España, de país de emirgantes a país de inmigrantes. Madrid: Ministerio de Trabajo y Asuntos Sociales e IMSERSO. http://umer.es/wpcontent/uploads/2015/05/n48.pdf

Adkins, M., Sample, B. y Birman, D. (1999). Mental Health and the Adult Refugee: The Role of the ESL Teacher. ERIC Digest. National Clearinghouse for ESL Literacy Education Washington DC.

APA (en línea). Post-traumatic stress disorder. Psychology Topics. American Psychological Association. Disponible en: www.apa.org/topics/ptsd/

APA (2011). El Camino a la Resiliencia. Disponible en: http://www.apa.org/centrodeapoyo/resiliencia-camino

Asensio Pastor M. I. (2016). La literatura en el proceso de inmersión lingüística por parte de alumnos inmigrantes. Revista Fuentes, 18(2), 197-208.

Asensio Pastor M. I. (2018). Enseñar español como Segunda Lengua a alumnos de Educación Infantil: retos y orientaciones metodológicas. Doblele: revista de lengua y literatura , 4, 108-121. https://doi.org/105565/rev/doblele.43

Asensio Pastor M. I. y Carmona García, J. P. (2019). Exploratory Research for the Improvement of the Teaching of Spanish as a Second Language in a Spanish Public Center. Languages, 4(4), 95. https://doi.org/10.3390/languages4040095

Badawi, E. M. (1973). Mustawayāt ${ }_{u}$ al-'arabiyat $_{i}$ al-mu'āṣirati fì miṣr. El Cairo: Dār alMa'ārif

Benseman, J. (2012). Adult refugee learners with limited literacy: needs and effective responses. AkoAotearoa. Disponible en: https://akoaotearoa.ac.nz/akohub/ako-aotearoa-northern-hub/adult-refugee-learners-Benseman

Bigelow, M., Vanek, M., King K. y N. Adi. (2017). Literacy as social (media) practice: Refugee youth and native language literacy at school. International Journal of Intercultural Relations, 60 , $183-197$. https://doi.org/10.1016/j.ijintrel.2017.04.002 
Blanc, H. (1960). Style variations in spoken Arabic: A simple of intedialectal educated conversation. En C. A. Ferguson (ed.). Contributions to Arabic Linguistics. (pp. 81-156). Harvard: Harvard University Press.

Bravo-García, E., Gallardo-Saborido, E., Santos de la Rosa, I. y Gutiérrez, A. (Eds.) (2014). Investigaciones sobre la enseñanza del español y su cultura en contextos de inmigración. Helsinki / Sevilla: Universidad de Helsinki / Universidad de Sevilla.

Calvo, J. (1999). Lingüística aplicada. En A. López (coord.) Lingüística General y Aplicada (pp. 323-348). Valencia: Univertitàt de Valencia.

Capstick, T. y Delaney, M. (2017). Language for resilience. The role of language in enhancing the resilience of Syrian refugees and host communities. En colaboración con la UNHCR. Disponible en: https://www.britishcouncil.org/sites/default/files/language-for-resiliencereport-en.pdf

Carlsson, J., Mortensen, E. y Kastrup, M. (2006). Predictors of mental health and quality of life in male and tortured refugees. Nordic Journal of Psychiatry, 60, 51-57. DOI: $10.1080 / 08039480500504982$

Carmona García, J.P. (2018). La enseñanza de E/LE a inmigrantes en las Escuelas Oficiales de Idiomas de Andalucía: Retos y propuestas. Doblele. Revista de lengua y literatura, 4: 122-39. https://doi.org/10.5565/rev/doblele.36

Carmona García, J. P. y García-Marcos, F. (2002). Contacto de lenguas y tipología lingüística en Almería. En J. D. Luque Durán, Pamies Bertrán y Manjón Pozas (eds.) Nuevas tendencias en la investigación lingüística (pp.67-79). Granada: Método.

Chadwik, A. y Awad, A. (2016). Language for Resilience: supporting Syrian refugees. British Council. Disponible en: https://www.britishcouncil.org/language-forresilience

Corvo, K., y Peterson, J. (2005). Post-traumatic stress, language acquisition, and selfsufficiency: a study of Bosnian refugees. Journal of Social Work, 5, 205-219. DOI: 10.1177/1468017305054974

Díaz-Corralejo Conde, J. (2001). Reflexiones sobre el interculturalismo en la enseñanza de las lenguas. Actas de las Jornadas sobre la enseñanza del español para inmigrantes y refugiados. Didáctica (Lengua y Literatura), 7, 321-331.

Elmeroth, E. (2011). From refugee camp to solitary confinement: illiterate adults learn Swedish as a second language. Scandinavian Journal of Educational Research, 47, 431-449. DOI: 10.1080/0031383032000100772

Ferguson, C. A. (1959). Diglossia. Word, $15 \quad$ (2), 325-340. https://doi.org/10.1080/00437956.1959.11659702 
Fernández García, F. (2009). Bases para la gestión de una situación de multilingüismo derivada de la inmigración. Cultura, lenguaje y representación (7), 59-76.

Feuernherm, E. y V. Ramanathan, (Eds.) (2015). Refugee resettlement in the United States: language, policy, pedagogy. Bristol: Multilingual Matters.

Finn, H. B. (2010). Overcoming barriers: Adult refugee trauma survivors in a learning community. TESOL Quarterly, 44, 586-596. DOI: 10.5054/tq.2010.232338

García-Marcos, F. (1999). Fundamentos críticos de sociolingüística. Almería: Universidad de Almería.

García-Marcos, F. (2002). Lenguaje e Inmigración (I). Sociolingüística e inmigración. Granada: Método.

García-Marcos, F. (2003). De la interculturalidad al mestizaje. Lenguas, culturas y conocimiento. En E. Soriano Ayala (Ed.) Diversidad étnica y cultural en las aulas (pp. 95-137). Madrid: La Muralla.

García-Marcos, F. (2005). Contacto y planificación lingüística en Almería. Language \& Design, (7), 85-105.

García-Marcos, F. (2017). Inmigración, contacto de lenguas y planificación social. RIEM Revista Internacional de Estudios Migratorios, I, 83-94. DOI: http://dx.doi.org/10.25115/riem.v1i1.369

García-Marcos, F. (2018). La trastienda de la enseñanza de lenguas extranjeras. Granada: Comares.

García-Mateos, C. (2004). Experiencias y propuestas para la enseñanza de L2 a personas inmigradas. Madrid: Edinumen.

García-Mateos, C. (2003). Experiencias y propuestas para la enseñanza de la L2 a personas inmigradas, Carabela, 53, 105-131.

García-Ortiz, E. M. y Khraiche Ruiz-Zorrilla, V. (2013). Un caso extremo de clase plurilingüe y heterogénea. Inmigrantes refugiados. Revista Nebrija de Lingüística Aplicada a la Enseñanza de Lenguas, 13.

García-Parejo, I. (2003). Los cursos de «español para inmigrantes» en el contexto de la educación de personas adultas. Carabela, 53, 45-64.

García-Parejo, I. (2004). La enseñanza del español a inmigrantes adultos. En Vademécum para la formación de profesores. Enseñanza española como segunda lengua (L2) /lengua extranjera (LE) (pp.1259-1277). Madrid: SGEL.

Gordon, D. M. (2011). Trauma and Second Language Learning among Laotian Refugees. Journal of Southeast Asian American Education \& Advancement.Vol. 6(1), 13.

Grognet, A. G. (1997). Elderly Refugees and Language Learning.Educational Resources Information Center (ERIC). Disponible en: https://files.eric.ed.gov/fulltext/ED416721.pdf 
Hayward, M. (2007). Applying Post-Critical Approaches to Refugees-Centred Education. (Tesis inédita). Disponible en: https://aut.researchgateway.ac.nz/bitstream/handle/10292/221/HaywardM.pd f?sequence

Hernández García, M. T. y Villalba Martínez, F. (2003a). La enseñanza de español a inmigrantes, ¿̇una metodología específica? En Actas del XII Encuentro Práctico de Profesores ELE, Barcelona, IH-Difusión.

Hernández García, M. T. y Villalba Martínez, F. (2003b). Análisis descriptivo de materiales didácticos para la enseñanza del español (L2) y la alfabetización de inmigrantes. La enseñanza del español como segunda lengua / lengua extranjera a inmigrantes, Carabela, 53, 133-160.

Herrera, F. (ed.) (2016). Enseñar español a niños y adolescentes. Enfoques y tendencias. Madrid: Difusión.

Instituto Cervantes (2005). Español como nueva lengua. Orientaciones para un curso de emergencia destinado a inmigrantes adultos. Madrid: Santillana. Universidad de Salamanca. Instituto Cervantes.

Jefatura del Estado (2009). Ley 12/2009 publicada en el BOE núm. 263 de 31 de octubre de 2009. https://www.boe.es/buscar/act.php?id=BOE-A-2009-17242

Jiménez Jiménez, A. (2015). Enseñar español sin recursos materiales. El caso de los refugiados saharauis. Educatio Siglo XXI, 33(2), 79-104. https://dx.doi.org/10.6018/i/232701

Keyes, E. F., y Kane, C. F. (2004). Belonging and adapting: mental health of Bosnian refugees living in the United States. Issues in Mental Health Nursing, 25, 80931. DOI: $10.1080 / 01612840490506392$

Kleinmann, H. H. (1984). Undertanding refugee Second Language Learning.JALT Journal, 6(2). https://jalt-publications.org/files/pdf-article/ij-6.2-art4.pdf

Larragaña Domínguez, A. (2003). Aproximación a una bibliografía sobre la enseñanza del español como segunda lengua / lengua extranjera a inmigrantes, Carabela, $53,161-176$.

Larsen-Freeman, D. y Long, M. H. (1991). Theories in second language acquisition. Londres: Longman. [1994] Introducción al estudio de la adquisición de segundas lenguas, Madrid: Gredos.

Mateo, M. V. (1995). Enseñanza de español a inmigrantes. Datos empíricos y propuestas teóricas. Revista de Estudios de Adquisición de la Lengua Española, 3, 177-127.

Martín Martín, J. M. (2004). La adquisición de la lengua materna (Ll) y el aprendizaje de una segunda lengua (L2) / lengua extranjera (LE): procesos cognitivos y factores condicionantes. En J. Sánchez Lobato e I. Santos Gargallo (eds.) 
Vademécum para la formación de profesores. Enseñar español como segunda lengua (L2) / Lengua extranjera (LE) (pp.261-286). Madrid: SGEL.

Ministerio de Empleo y Seguridad Social. Centro de Acogida a Refugiados (CAR). Disponible en: https://extranjeros.empleo.gob.es/es/ProteccionAsilo/car/

Ministerio del Interior (en línea). Datos e información estadística. Disponible en: http://www.interior.gob.es/web/servicios-al-ciudadano/oficina-de-asilo-yrefugio/datos-e-informacion-estadistica\#\%C3\%BAltimo\%20datos

Ministerio de la Presidencia (2011). Real Decreto 557/2011, de 20 de abril, por el que se aprueba el Reglamento de la Ley Orgánica 4/200o, sobre derechos y libertades de los extranjeros en España y su integración social, tras su reforma por Ley Orgánica 2/2009. Disponible en: BOE-A-2011-7703

Miquel López, L. (1994). Enseñanza del español como lengua extranjera a inmigrantes y refugiados. En Emigración y refugio. Primeras Jornadas Educativas en Móstoles.

Miquel López, L. (1995). Reflexiones previas sobre la enseñanza de E/LE a inmigrantes y refugiados. Didáctica. Lengua y Literatura, 7, 241-270.

Muñoz, C. (2002). Aprender idiomas. Barcelona, Paidós.

Muñoz López, B. (2004). La enseñanza del español a inmigrantes en el marco institucional. En J. Sánchez Lobato e I. Santos Gargallo (Eds.) Vademécum para la formación de profesores. Enseñar español como segunda lengua (L2) / Lengua extranjera (LE) (pp.1205-1223). Madrid: SGEL.

Naeb, R., Young-Scholten, M. y Sosinski, M. (2019). Immigrant and Refugee Languages. Languages, 4(3). Disponible en: https://www.mdpi.com/2226-471X/4/3

Níkleva, D. G. (coord.) (2014). El reto de atender a alumnos inmigrantes en la sociedad española. Madrid: Síntesis.

Níkleva, D. G. (ed.) (2017). La formación de los docentes de español para inmigrantes en distintos contextos educativos. Bern: Peter Lang.

Nussbaum, L. y Unamuno, V. (2014). Luces y sobras de la educación plurilingüe en España y América Latina. En C. Lomas (Ed.) La educación lingüística, entre el deseo y la realidad (pp. 203-215). Barcelona: Octaedro.

Organización Internacional para las Migraciones. (2013). Informe sobre las migraciones en el mundo 2013. Disponible en:

http://publications.iom.int/system/files/pdf/wmr2013 sp.pdf

OIM (2019). Informe sobre las Migraciones en el Mundo 2020. Disponible en: https://publications.iom.int/system/files/pdf/wmr_2020_es.pdf 
Organización de las Naciones Unidas para la Educación, la Ciencia y la Cultura. (2017). Tercer informe mundial sobre el aprendizaje y la educación de adultos. Disponible en: https://unesdoc.unesco.org/images/0024/002475/247556s.pdf

Ojeda Álvarez, D. (2009). Lenguas y culturas en la escuela: cómo organizarlas. En A. Ríos Rojas y G. Ruiz Fajardo (Eds.) Didáctica del español como $2^{a}$ lengua para inmigrantes (pp.80-91). Sevilla: Universidad Internacional de Andalucía (UNIA).

ONU (1951). Convenio sobre el Estatuto de los Refugiados. Disponible en: https://www.acnur.org/fileadmin/scripts/doc.php?file=fileadmin/Documentos $\angle \mathrm{BDL} / 2001 / 0005$

ONU (1967). Protocolo sobre el Estatuto de los Refugiados. Disponible en:

https://www.acnur.org/fileadmin/scripts/doc.php?file=fileadmin/Documentos /BDL/2001/0003

ONU (12 de enero de 2016). ONU cifra en 244 millones los migrantes que vivien fuera de su país. Noticias. Departamento de Asuntos Económicos y Sociales. https://www.un.org/development/desa/es/news/population/internationalmigrants.html

Posada, P. A. (2009). Refugiados y desplazados forzados. Categorías de la migraión forzada creadas como medidas de contención a las migraciones no deseadas. Estudios Poñlíticos, $\mathrm{n}^{\mathrm{o}} 35$

Pozzo, M. I. (Ed.) (2009). Migraciones y formación docente. Aportes para una educación intercultural. Bern: Peter Lang.

Raga, F. y Ortí, R. (1997). Problemas teóricos y prácticos de la enseñanza del español a inmigrantes centroafricanos. En C. Hernández Sacristán y R. Morant Marco (Eds.) Lenguaje y Emigración (175-187). Valencia: Universitat de València.

Richards, J. C. y Rodgers, T. S. ([1986] 1998). Enfoques y métodos en la enseñanza de idiomas. Madrid: Cambridge UniversityPress.

Salazar García, V. (1998). La enseñanza del español a inmigrantes senegaleses: la experiencia de ADESEAN. Revista de Humanidades y Ciencias sociales del IEA, $16,237-250$.

Salazar García, V. (2004). Adquisición de segundas lenguas. Granada. Método.

Santos Gargallo, I. (1999). Lingüística aplicada a la enseñanza-aprendizaje del español como lengua extranjera. Madrid: Arco/Libros.

Simas, H., Halat, R., Maayeh, R., Dabdoub, R., Rafidi, T., AbouShaaban, S. y Hamdy, H. (2017). Blended English Language Learning for Refugees: Challenges and Possibilities.Cambridge: CUP. 
Slagter, P. J. (1994). Fiabilidad y validez en tests de lengua. A propósito de un test de elección múltiple de español como lengua extranjera. REALE, 1, 137-156.

Spindler, W. (2015). El año de la crisis de refugiados en Europa. Blog UNHCR/ACNUR. La Agencia de la ONU para los Refugiados. España.

Utria Utria, L., Amar Amar, J., Martínez González, M., Colmenares López, G. y Crespo Romero, F. (2015). Resiliencia en mujeres víctimas del desplazamiento forzado. Barranquilla: Universidad del Norte Editorial y Uniediciones.

Valero-Matas, J, Coca, J. R. y Valero-Oteo. (2014). Análisis de la inmigración en España y la crisis económica. Papeles de población, 20(80), 9-45.

Villalba Martínez, F. y Hernández García, M. T. (1995). Las clases de lengua y cultura para inmigrantes y refugiados. Didáctica. Lengua y Literatura, 7, 425-432.

Villalba García, F. y Hernández Martínez, M. T. (2000). La enseñanza de español a inmigrantes y refugiados adultos. En Actas de la II Escuela de Verano. Metodología y Evaluación de Personas Adultas. Madrid: Comunidad de Madrid, 97-118.

Villalba García, F. y Hernández Martínez, M. T. (2002). La enseñanza del español como segunda lengua a inmigrantes y refugiados: formación del profesorado. Cuadernos de bitácora, 4 39-48. https://www.redined.mepsyd.es/xmlui/handle/11162/33082

Villalba García, F. y Hernández Martínez, M. T. (2010). Perspectivas y líneas de trabajo en la enseñanza de español a inmigrantes. MarcoELE, 10, 161-184.

Villalba Martínez, F., Hernández García, M. T. y Aguirre, C. (2001). Orientaciones para la enseñanza del español a inmigrantes y refugiados. Madrid: Ministerio de Educación $\quad$ y $\quad$ Ciencia. Disponible https://sede.educacion.gob.es/publiventa/orientaciones-para-la-ensenanzadel-espanol-a-inmigrantes-y-refugiados/ensenanza-lengua-espanola/7747

Villarrubia Zúñiga, M. (2009). El aprendizaje de ELE a niños y adolescentes inmigrantes. marcoELE. Revista de Didáctica Español Lengua Extranjera, (8), 1-15. 